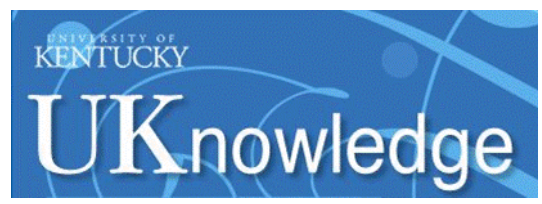

University of Kentucky

UKnowledge

2-21-2018

\title{
Effect of Molecular Side Groups and Local Nanoenvironment on Photodegradation and Its Reversibility
}

Nicole Quist

Oregon State University

Mark Li

Oregon State University

Ryan Tollefsen

Oregon State University

Michael Haley

University of Oregon

John Anthony

University of Kentucky, anthony@uky.edu

See next page for additional authors

Follow this and additional works at: https://uknowledge.uky.edu/chemistry_facpub

Part of the Chemistry Commons, Electronic Devices and Semiconductor Manufacturing Commons, and the Semiconductor and Optical Materials Commons

Right click to open a feedback form in a new tab to let us know how this document benefits you.

\section{Repository Citation}

Quist, Nicole; Li, Mark; Tollefsen, Ryan; Haley, Michael; Anthony, John; and Ostroverkhova, Oksana, "Effect of Molecular Side Groups and Local Nanoenvironment on Photodegradation and Its Reversibility" (2018). Chemistry Faculty Publications. 135.

https://uknowledge.uky.edu/chemistry_facpub/135

This Conference Proceeding is brought to you for free and open access by the Chemistry at UKnowledge. It has been accepted for inclusion in Chemistry Faculty Publications by an authorized administrator of UKnowledge. For more information, please contact UKnowledge@lsv.uky.edu. 


\title{
Effect of Molecular Side Groups and Local Nanoenvironment on Photodegradation and Its Reversibility
}

\author{
Digital Object Identifier (DOI) \\ https://doi.org/10.1117/12.2291065
}

\section{Notes/Citation Information}

Published in Proceedings of SPIE, v. 10529, Organic Photonic Materials and Devices XX, article 105290Z, p. 1-9.

(C) 2018 SPIE. One print or electronic copy may be made for personal use only. Systematic reproduction and distribution, duplication of any material in this publication for a fee or for commercial purposes, and modification of the contents of the publication are prohibited.

Nicole Quist, Mark Li, Ryan Tollefsen, Michael Haley, John Anthony, and Oksana Ostroverkhova, "Effect of molecular side groups and local nanoenvironment on photodegradation and its reversibility," Proc. SPIE 10529, Organic Photonic Materials and Devices XX, $105290 Z$ (February 21, 2018). DOI: https://doi.org/ $10.1117 / 12.2291065$

The copyright holder has granted the permission for posting the article here.

\section{Authors}

Nicole Quist, Mark Li, Ryan Tollefsen, Michael Haley, John Anthony, and Oksana Ostroverkhova 


\section{Effect of molecular side groups and local nanoenvironment on photodegradation and its reversibility}

Nicole Quist, Mark Li, Ryan Tollefsen, Michael Haley, John Anthony, et al.

Nicole Quist, Mark Li, Ryan Tollefsen, Michael Haley, John Anthony, Oksana Ostroverkhova, "Effect of molecular side groups and local nanoenvironment on photodegradation and its reversibility," Proc. SPIE 10529, Organic Photonic Materials and Devices XX, 105290Z (21 February 2018); doi: 10.1117/12.2291065

SPIE. Event: SPIE OPTO, 2018, San Francisco, California, United States 


\title{
Effect of molecular side groups and local nanoenvironment on photodegradation and its reversibility
}

\author{
Nicole Quist ${ }^{\mathrm{a}}$, Mark Li ${ }^{\mathrm{a}}$, Ryan Tollefsen ${ }^{\mathrm{a}}$, Michael Haley ${ }^{\mathrm{b}}$, John Anthony ${ }^{\mathrm{c}}$, and Oksana \\ Ostroverkhova ${ }^{b}$ \\ ${ }^{a}$ Oregon State University, Corvallis, Oregon, United States \\ ${ }^{\mathrm{b}}$ University of Oregon, Eugene, Oregon, United States \\ ${ }^{c}$ University of Kentucky, Lexington, Kentucky, United States
}

\begin{abstract}
Degradation of organic semiconductors in the presence of oxygen is one of the bottlenecks preventing their wide-spread use in optoelectronic devices. The first step towards such degradation in functionalized pentacene $(\mathrm{Pn})$ derivatives is formation of endoperoxide (EPO), which can either revert back to the parent molecule or proceed to molecule decomposition. We present the study of reversibility of EPO formation through probing the photophysical properties of functionalized fluorinated pentacene (Pn-R-F8) derivatives. Experiments are done in solutions and in films both at the single molecule level and in the bulk. In solutions, degradation of optical absorption and its partial recovery after thermolysis were quantified for various derivatives depending on the solvent. At the single molecule level, low concentrations of each type of molecules were imaged in a variety of polymer matrices at $633 \mathrm{~nm}$ excitation at room temperature in air using wide-field fluorescence microscopy. Fluorescence time trajectories were collected and statistically analyzed to quantify blinking due to reversible EPO formation depending on the host matrix. To understand the physical changes of the molecular system, a Monte Carlo method was used to create a multi-level simulation, which enabled us to relate the change in the molecular transition rates to the experimentally measured parameters. At the bulk level, photoluminescence decay due to photobleaching and recovery due to EPO reconversion were measured for the same derivatives incorporated into various matrices. These studies provide insight into the synergistic effect of the local nanoenvironment and molecular side groups on the oxygen-related degradation and subsequent recovery which is important for development of organic electronic devices.
\end{abstract}

Keywords: Organic semiconductors, photodegradation, thermolysis, single molecule fluorescence spectroscopy, Monte Carlo, fluorescence intermittency

\section{INTRODUCTION}

The use of organic materials in low-cost (opto)electronic and photonic devices is of substantial current interest. ${ }^{1}$ Regardless of the application, successful commercialization of devices is hindered by the degradation of the organic materials due to reactions with oxygen, water, and so on. Previous studies have indicated that exposure to oxygen is detrimental to the lifetime of promising organic molecules exemplified by acene derivatives, in which the dominant oxygen-induced degradation mechanism is the formation of endoperoxides (EPO). ${ }^{2,3}$ However, EPO formation rate is environment dependent, and can be manipulated with the change in the film composition. ${ }^{3}$ To better understand which environmental changes are most effective in inhibiting EPO formation and in prolonging the lifetime of devices, we study these changes on both the nanoscale with single molecule spectroscopy ${ }^{3,4}$ and on the semi-bulk scale with absorption and photoluminescence (PL) measurements of dilute solutions and films containing low concentrations of molecules of interest incorporated into polymer matrices. ${ }^{5,6}$ Our studies probe the photophysics of technologically relevant solution-processable high-mobility functionalized acene derivatives which have been previously utilized in thin-film-transistors and other (opto)electronic applications. ${ }^{1,7}$

Further author information: (Send correspondence to O.O.)

E-mail: oksana@science.oregonstate.edu, Telephone: 15417371679

Organic Photonic Materials and Devices XX, edited by Christopher E. Tabor, François Kajzar,

Toshikuni Kaino, Yasuhiro Koike, Proc. of SPIE Vol. 10529, 105290Z · @ 2018 SPIE

CCC code: $0277-786 \mathrm{X} / 18 / \$ 18 \cdot$ doi: $10.1117 / 12.2291065$

Proc. of SPIE Vol. 10529 105290Z-1 
On the single-molecule level, it has been established that fluorescence intermittency (blinking) observed in Pn-R-F8 molecules incorporated in a polymer matrix is related to reversible EPO formation. ${ }^{3}$ However, understanding of energy and charge transfer processes between the photoexcited molecule and other molecules in the surrounding environment during the molecule degradation and recovery is difficult due to complexity of the nanoscale environment. ${ }^{8-11}$ To aid in the interpretation of the data, Monte Carlo simulations have been utilized to model fluorescence time trajectories that are affected by these processes. ${ }^{12-15}$ In particular, we seek to further refine the model and to determine the accuracy of the single molecule analysis used in experimental data processing. Through both experimental and computational approaches, we aim to better understand the requirements for molecular structure and local environment that enable stable organic (opto)electronic devices less susceptible to photodegradation.

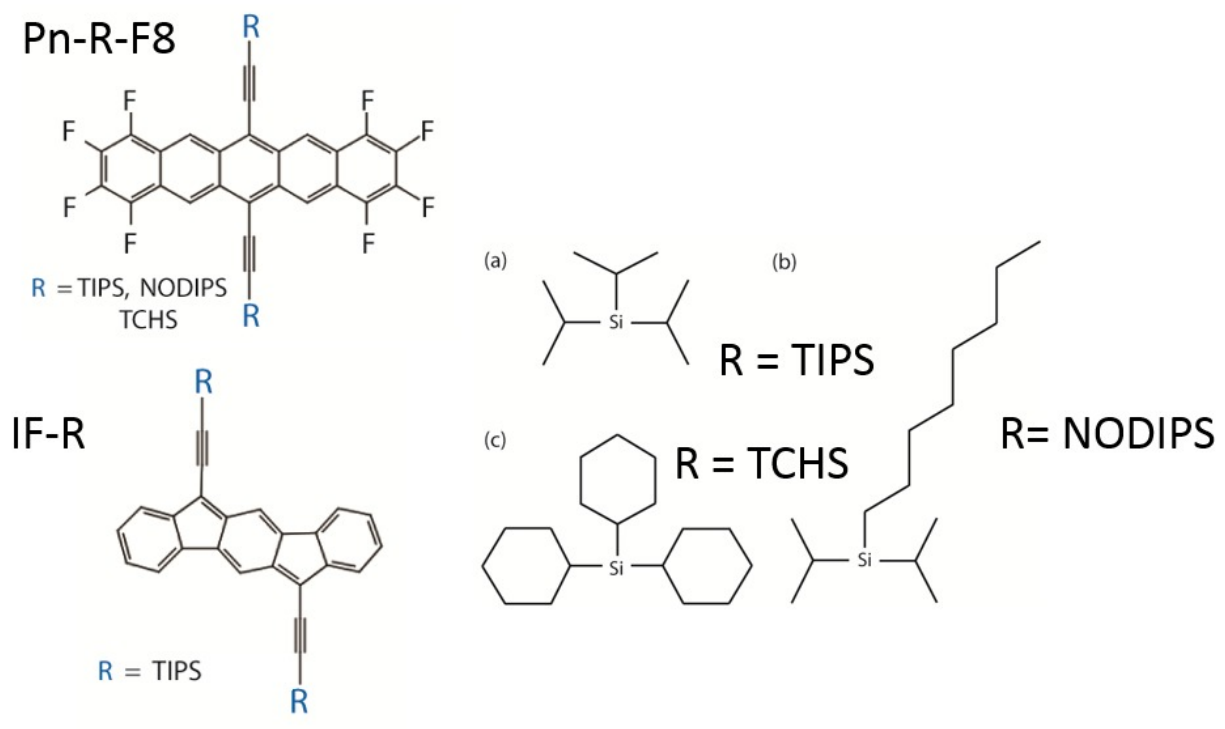

Figure 1. Functionalized pentacene (Pn-R-F8) and indenofluorene (IF-R) derivatives used in our studies. Structures of the side groups $\mathrm{R}$ are also included.

\section{EXPERIMENTAL}

\subsection{Materials}

In order to investigate the effects of the side groups and host matrix on photodegradation, we chose three fluorinated pentacene (Pn) derivatives (Pn-R-F8) with functionalized side groups R of TCHS ((tricyclohexylsilyl)ethynyl), NODIPS ((n-octyldiisopropylsilyl)ethynyl), and TIPS ((triisopropylsilylethinyl)) shown in Figure 1. These derivatives share similar absorption and PL spectra in isolated molecules, but their intermolecular interactions, as well as interactions with the environment, depend on the side group R. ${ }^{7}$ These molecules have been previously utilized as acceptors in donor-acceptor heterojunctions, exhibiting strong dependence of exciton and charge carrier dynamics on the size of the R group (TCHS > NODIPS $>$ TIPS). ${ }^{7,16,17}$ Additionally, reversible EPO formation has been observed on the single-molecule level in Pn-TCHS-F8 molecules incorporated in poly(methyl)methacrylate (PMMA). ${ }^{3}$ Moreover, this process could be controlled by the addition of a different type of molecule, such as an indenofluorene derivative (IF-TIPS in Figure 1) to the PMMA matrix.

For studies in solution, Pn-R-F8 molecules were dissolved either in benzene or in chlorobenzene (CB) at a concentration of $2 \times 10^{-5} \mathrm{M}$. The semi-bulk thin film preparation involved combining $30 \mathrm{mM}$ Pn-R-F8 solutions in toluene with a $13 \mathrm{wt} \%$ PMMA solution in toluene at appropriate ratios $^{3,5,6}$ to yield average Pn-Pn intermolecular spacings in the 3-11 $\mathrm{nm}$ range. In selected cases, IF-TIPS molecules (1 $\mathrm{mM}$ solution in toluene) were added to the mixture at concentrations that would achieve the average IF-IF intermolecular spacing of $5 \mathrm{~nm}$ in the film. After a 20 minute sonication of mixtures of solutions, the films (either Pn-R-F8:PMMA or Pn-R-F8:IF-TIPS:PMMA) 
were deposited by spin-casting at $3000 \mathrm{rpm}$ for 50 seconds from $60 \mu \mathrm{L}$ of solution. For studies at the singlemolecule level, ultra-low $\left(10^{-10} \mathrm{M}\right)$ concentrations of Pn-TCHS-F8 molecules were incorporated in PMMA (Mw $75,000 \mathrm{~g} / \mathrm{mol}$ ), polystyrene (PS, Mw 280,000 g/mol), or poly(9-vinyl)carbazole (PVK, Mw 1,100,000 g/mol) matrices as described in our previous publications. ${ }^{3,4}$

\subsection{Measurements of optical absorption and photoluminescence}

The propensity of Pn-R-F8 molecules for photodegradation in solutions was measured by monitoring optical absorption of solutions irradiated by a UV lamp $(300-400 \mathrm{~nm})$ at $0.5 \mathrm{~mW} / \mathrm{cm}^{2}$ in air for up to 100 hours, depending on the solvent. Absorption spectra was taken using a xenon lamp illumination of the solution through a $200 \mu \mathrm{m}$ optical fiber and collecting the transmitted light through a $50 \mu \mathrm{m}$ optical fiber which was processed by the Ocean Optics USB2000 spectrometer. Spectrum of the solvent without Pn-R-F8 molecules served as reference.

Once the solution was completely photobleached so that no absorption in the 500-700 $\mathrm{nm}$ region (Fig. 2 (a) ) could be detected, the sample went through thermolysis. For this, the vial was sealed, wrapped in foil and submerged in water at $80^{\circ} \mathrm{C}$ for two sets of three hours, with absorption data collected at the end of each three hour set (Fig. 2 (a)).

To measure the PL decay due to photobleaching in semi-bulk films, an Olympus IX-71 inverted microscope was used with a 10x magnification objective lens, which focused a $633 \mathrm{~nm}$ HeNe laser with power ranging between 300 and $700 \mu \mathrm{W}$ onto a prepared sample. The PL was collected through a $600 \mu \mathrm{m}$ optical fiber and processed by an Ocean Optics USB2000-FLG spectrometer. Six hundred consecutive spectra were collected with a integration time of 100 milliseconds and 5 scans to average. A dark spectrum was also taken as a background reference, and this was subtracted from the data. Each spectrum was integrated over the 500-700 nm region of interest, which provided a value for each respective time. For studies of photobleaching reversibility, after the first experimental run, the sample was kept in the dark for 20 minutes at room temperature in air, after which the experiment was repeated (run 2) on the same sample area.

Single molecule fluorescence spectroscopy was performed under a $633 \mathrm{~nm}$ excitation, using a 100x objective and Olympus IX-71 inverted microscope, in the wide-field geometry with an EMCCD detector, as described in detail in our previous publications. ${ }^{3,4}$

\section{RESULTS AND DISCUSSION}

\subsection{Photodegradation and recovery in solution}

Figure 2 shows (a) how photodegradation of Pn-R-F8 molecules manifests into optical absorption spectra and (b) how the polarity of the solvent affects the rate of the degradation. Upon prolonged UV irradiation, the 500-700 $\mathrm{nm}$ absorption band (corresponding to the $\mathrm{S}_{0}-\mathrm{S}_{1}$ transition in Pn-R-F8 molecules) disappears, and an enhanced absorption in the 350-450 nm region is observed. Similar effect was previously observed upon degradation of nonfluorinated functionalized Pn derivatives (such as Pn-TIPS) and attributed to the photodimerization ${ }^{18}$ and EPO formation. ${ }^{2}$ In the fluorinated derivatives, Pn-R-F8, used in our studies, both processes should be considerably less pronounced, as indeed can be seen from the comparison of the Pn-R-F8 versus Pn-TIPS data in benzene in Fig. 2(b). In particular, in benzene the half-lifetime (defined as the time over which the PL has decayed to the $50 \%$ level as compared to that in the fresh sample at $t=0$ ) of Pn-TIPS-F8 derivative is about a factor of 4 higher than that of Pn-TIPS, consistent with our previous observations of higher stability of Pn-TIPS-F8 as compared to Pn-TIPS when incorporated into PMMA. ${ }^{4}$

The Pn-R-F8 derivatives exhibit more than an order of magnitude (a factor of 15-40, depending on the derivative) higher stability in benzene as compared to CB (Fig. 2(b)). A strong dependence of photodegradation on the solvent polarity, and the case of benzene versus CB in particular, was also observed for Pn-TIPS ${ }^{18}$ and attributed to the $\pi-\pi$ interactions of the Pn molecule with the surrounding benzene molecules which prevented Pn-Pn dimerization. Similar considerations can be applicable to the case of fluorinated Pn derivatives, although quantitative assessment of relative contributions of photodimerization and EPO formation into degradation of Pn-R-F8 in solution requires further studies. 
(a)

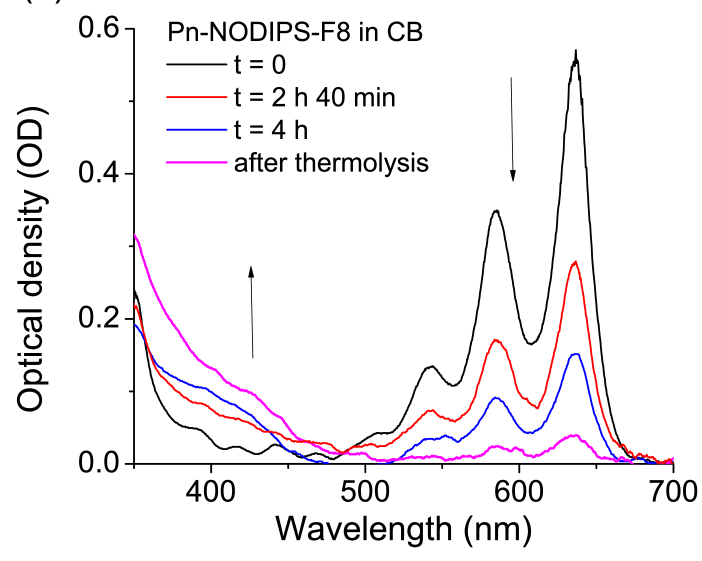

(b)

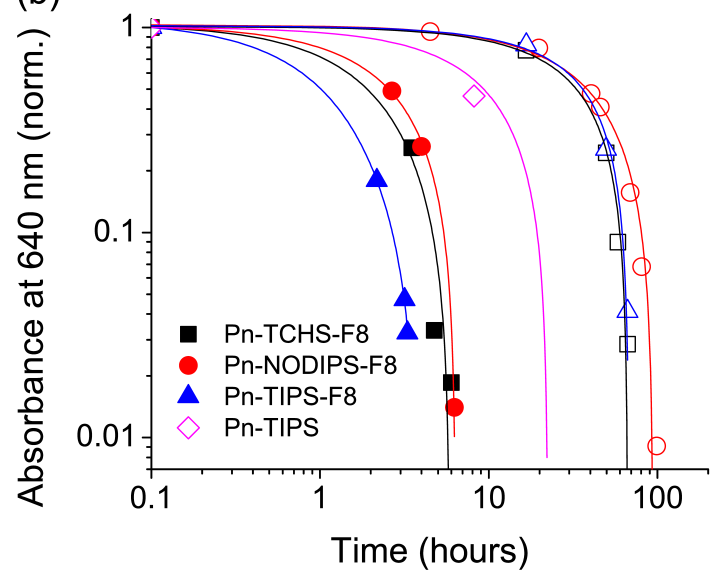

Figure 2. (a) Degradation under UV illumination over time $\mathrm{t}$ and partial recovery of the optical absorption spectra after 6 hours of thermolysis of Pn-NODIPS-F8 solution in chlorobenzene (CB). No absorption in the 500-700 nm region was observed after 7 hours of UV illumination at which point the thermolysis was initiated. (b) Absorbance at $640 \mathrm{~nm}$ normalized by its value at $\mathrm{t}=0$ (fresh solution) at various UV irradiation times obtained in Pn-R-F8 solutions in benzene (open) and CB (solid). Data for Pn-TIPS in benzene are also included.

The effect of side groups $\mathrm{R}$ on the photobleaching of Pn-R-F8 in solution was more pronounced in $\mathrm{CB}$ as compared to benzene (Fig. 2(b)), with TCHS and NODIPS derivatives exhibiting about a factor of 2 longer half-lifetimes than the TIPS derivative. This is consistent with our previous observations of higher stability of NODIPS and TCHS derivatives as compared to TIPS, in Pn-R-F8:PMMA films, ${ }^{4}$ indicative of a protective role of large side groups against photo-induced degradation.

In both solvents, and for all derivatives, the 3-6 hour thermolysis recovered about $6 \%$ of the original 500-700 $\mathrm{nm}$ absorption strength observed in fresh solutions. This recovery is most likely due to the EPO converting to the parent Pn molecules, as reversal of photodimerization would not be expected under low-temperature thermolysis conditions used in our experiments. The percentage of recovery did not considerably change between 3 and 6 hours of thermolysis; however, the absorption spectra obtained after 6 hours of thermolysis had an increased 350-450 $\mathrm{nm}$ spectral component as compared to those after 3 hours, which indicates that degradation processes continue during thermolysis.

\subsection{Photodegradation and recovery in films}

Figure 3 shows photobleaching of Pn-TCHS-F8 molecules incorporated in PMMA, measured via monitoring PL under continuous $633 \mathrm{~nm}$ illumination over $>600 \mathrm{sec}$, depending on the Pn concentration (a) and on the local environment (b-c). In particular, samples with higher concentrations of Pn-TCHS-F8 exhibited better photostability, most likely due to formation of nanoaggregates with reduced free volume, which decreased the oxygen permeability resulting in inhibited reactions of Pn-TCHS-F8 molecule with oxygen. After photobleaching the Pn-TCHS-F8:PMMA sample for 10 min, about a 5\% recovery in PL was observed after the sample was kept in the dark for 20 min (Fig. 3(b)) due to the EPO conversion to the parent Pn-TCHS-F8 molecule. The percentage of recovery under the same conditions doubled when IF-TIPS molecules were introduced in the film (i.e. in Pn-TCHS-F8:IF-TIPS:PMMA films, Fig. 3(c)) due to IF-TIPS molecules acting as quenchers of the highly reactive singlet oxygen produced upon the conversion of the EPO to the parent Pn-TCHS-F8 molecule,,$^{2,3}$ thus preventing an immediate attack of the newly recovered Pn-TCHS-F8 molecule. This effect was also observed at the single-molecule level. ${ }^{3}$ 

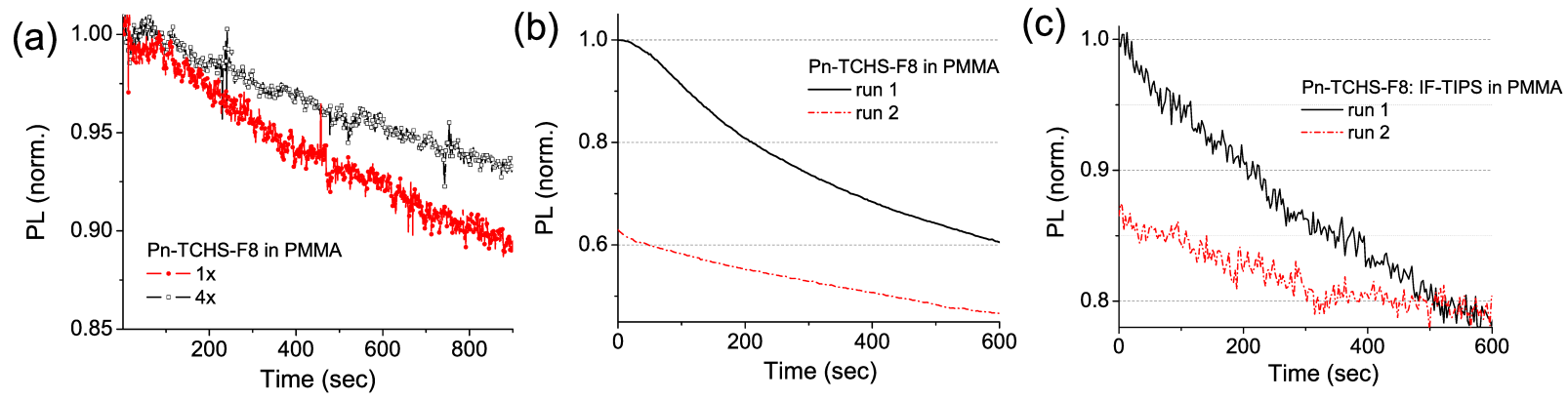

Figure 3. (a) The integrated PL spectra in the 500-700 $\mathrm{nm}$ region showing decay due to photodegradation under continuous $633 \mathrm{~nm}$ illumination of Pn-TCHS-F8:PMMA films at Pn-TCHS-F8 concentrations which differ by a factor of 4. (b) and (c) The integrated PL spectra versus time for Pn-TCHS-F8:PMMA and Pn-TCHS-F8:IF-TIPS:PMMA, respectively. Run 2 corresponds to the data from the same sample area obtained after the sample was kept in the dark in air for $20 \mathrm{~min}$ at room temperature.

\subsection{Monte Carlo simulations of single-molecule fluorescence time trajectories}

\subsubsection{The model}

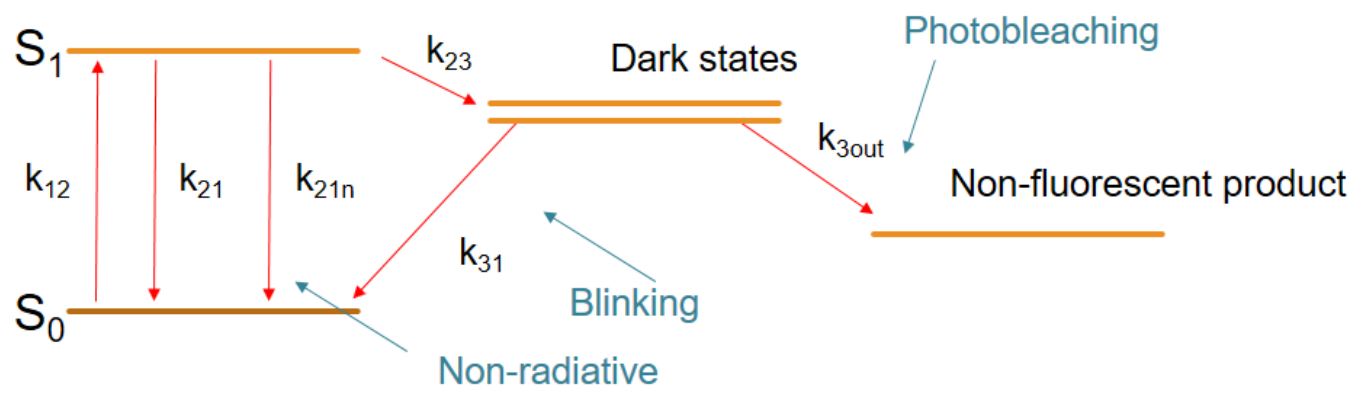

Figure 4. The four-level system that includes the ground state, excited state, long-lived (reversible) dark state, and a photobleached (irreversible) dark state. Rates incorporated in the model are also included.

In preparation for single molecule florescence spectroscopy, we refined the Monte Carlo simulation, which models the fluorescence time trajectories of individual molecules. The fluorescence counts obtained from each single molecule as a function of time are processed to produce time traces similar to those of Fig. 5(a), from which "on" and "off" times can be extracted and statistically analyzed as described in our previous publication. ${ }^{3}$ The complementary cumulative distribution functions (CCDFs) are then created for these "on" and "off" times (e.g. Fig. 5(b)) and fit to known distributions such as Weibull, exponential and lognormal using maximum likelihood estimation (MLE). The Monte Carlo simulation creates similar fluorescence time traces (Fig. 5(a)), which can be processed identically to the experimental data. By manipulating the parameters of the simulation we can mimic the experimental changes which provides key insights into the photophysical processes under study.

Previous research has modeled the charge carrier dynamics as observed in single-molecule fluorescence experiments by using a three-level system, with a ground state, excited state and dark state. ${ }^{12-15}$ Our previous work has used a four-level system, which includes the states mentioned above and, additionally, an irreversible "photobleached" state, as well as a non-radiative recombination rate $\left(k_{21 n}\right)$ between the excited state and the ground state (Fig. 4). In this model, the states 1 and 2 are the ground and excited states of the Pn-R-F8 molecule, whereas dark state 3 is the EPO obtained as a result of the reaction of Pn-R-F8 molecule with singlet 
oxygen. When the EPO reverts to the parent Pn-R-F8 molecule, the molecule can be re-excited again ("blinking"). ${ }^{3}$ The state 4 is a decomposed molecule. Using known experimental parameters, such as light intensity and fluorescence quantum yield of Pn-R-F8 molecules under study, ${ }^{4}$ we calculated realistic rates for $k_{12}, k_{21}$, and $k_{21 n} \cdot{ }^{19}$ In choosing realistic values for $k_{23}, k_{31}$ and $k_{3 o u t}$, we focused on rates that produce numerically simulated data similar to the experimental data and that result in a range of "on" and "off" times that produce fits with the highest accuracy. For time traces of $100 \mathrm{sec}$ duration typically used in our experiments and thus used in simulations, it is important that the average "on" and "off" times are considerably shorter than the 100 sec time window and yet long enough to be experimentally realistic. Modifying these values and incorporating distribution rates for the transitions provide significant insight into the accuracy of the data processing for the experimental data.
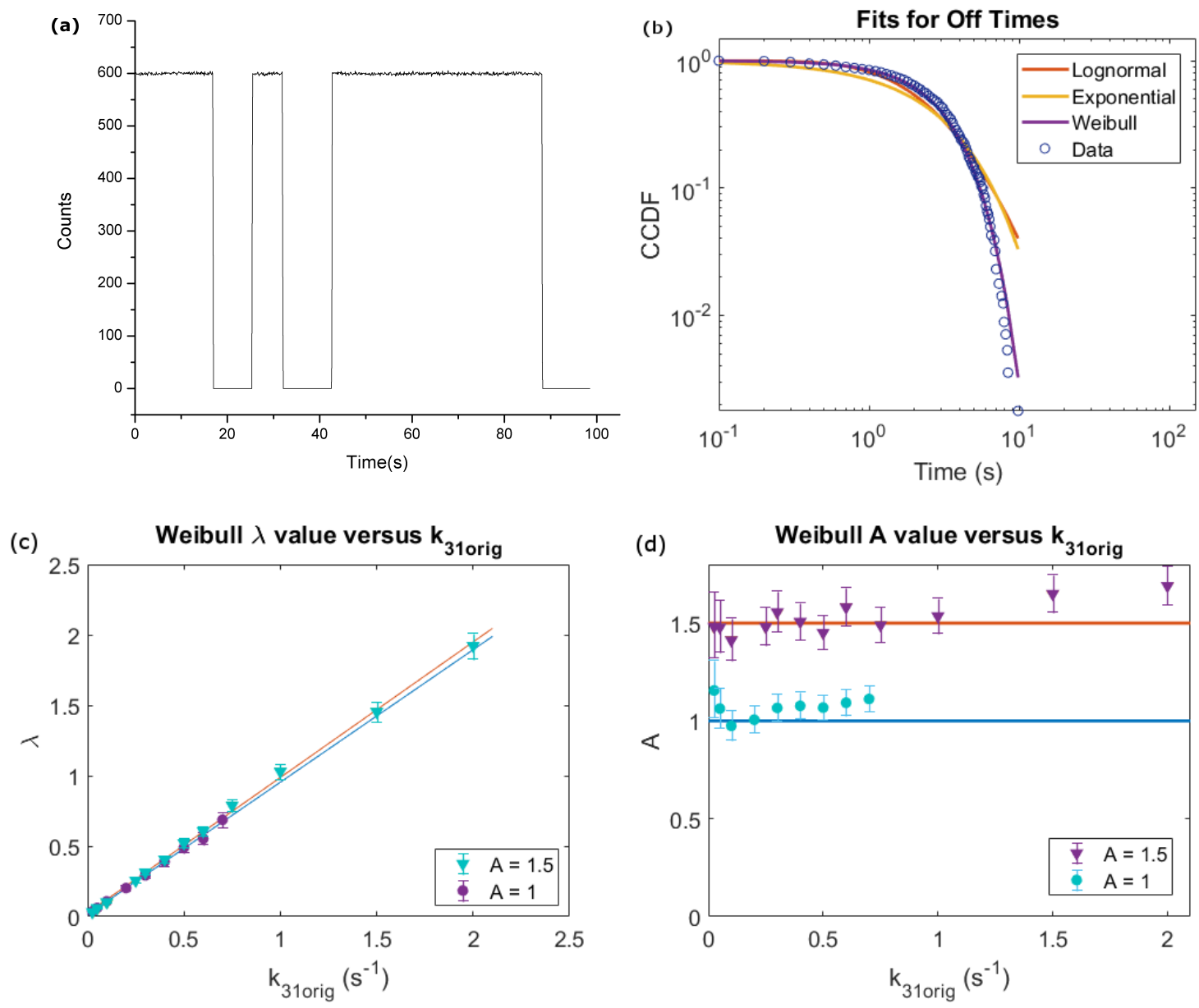

Figure 5. The Monte Carlo Simulation produces time traces (a) as outputs for the data which are converted to CCDFs (b) and fit to a variety of distributions. (c) and (d) Weibull fit values of "off" times compared to the variation in the $k_{31 \text { orig }}$ rate, with $A=1$ or $A=1.5$ and $k_{12}=2.9 \times 10^{4} s^{-1}, k_{21}=7 \times 10^{7} s^{-1}, k_{23}=222 s^{-1}$, and $k_{21 n} \approx 1.5 \times 10^{7} s^{-1}$. The $A$ fit parameters lie close to their true values and the fits for the $\lambda$ values are linear with respect to $k_{31 \text { orig }}$ with the slope close to 1 , as expected. In particular, $\lambda=0.94 k_{31 \text { orig }}+0.01$ for $A=1$ and $\lambda=0.96 k_{31 \text { orig }}+0.02$ for $A=1.5$.

The initial results from the simulation show that the "on" times for the simulation are affected by the $k_{23}$ rate and the "off" times are affected by both the $k_{31}$ and the $k_{3 o u t}$ rates. It was also seen that when the rates were fixed, the data fit an exponential distribution. ${ }^{19}$ Since the experimental data are typically described by a more complicated distribution such as Weibull distribution, this indicates a variation in the transition rates with respect to time. ${ }^{3}$ In particular, the Weibull-distributed process is characterized by a time-dependent rate 
$k(t)=(A / \lambda)(t / \lambda)^{A-1}$, where $A$ and $\lambda$ are Weibull fit parameters. ${ }^{20}$ (If $A=1$, the time-independent rate $k=1 / \lambda$, characteristic of the single-exponential distribution, is recovered.)

To investigate how such time-dependent rate manifests into the CCDF so that such rate can be successfully recovered from CCDF fits of the experimental data, we performed Monte Carlo simulations as follows. The model was simplified down to three states (1-3). The rates $k_{12}, k_{21}$, and $k_{23}$ were fixed at $2.9 \times 10^{4}, 7 \times 10^{7}$, and 222 , respectively. The rate $k_{21 n}$ was fixed such that $k_{21} /\left(k_{21}+k_{21 n}+k_{23}\right)=0.82$, where 0.82 is the quantum yield of Pn-R-F8 molecules under study, resulting in $k_{21 n}$ approximately equal to $1.5 \times 10^{7}$. The Weibull rate $k(t)$ was applied to the $k_{31}$ rate so that $k_{31}=\left(A * k_{31 \text { orig }}\right)\left(k_{31 \text { orig }} * t\right)^{A-1}$, where $k_{31 \text { orig }}$ is the original rate, which was varied from $0.025-2, t$ is the time the molecule has spent in the dark state (state 3 ), and $A$ is the Weibull distribution parameter, which for our experiments varied from $1.2-1.5 .^{3}$ From these rates the predicted average "on" time can be calculated using $\tau_{o n}=\left(k_{21}+k_{21 n}+k_{23}\right) /\left(k_{23} * k_{12}\right)$, which is 13.15 seconds. The predicted average "off" time is calculated using $\tau_{\text {off }}=\left(1 / k_{31 \text { orig }}\right) * \Gamma(1+1 / A)$, where $\Gamma$ is the Gamma function. For the range of $k_{31 \text { orig }}$ used in our simulations the predicted average "off" times range from $0.45-36.1$ seconds for $A=1.5$ and $1.4-40$ for $A=1$. Even at the high end, the sum of the predicted average "on" times and "off" time is lower than the $100 \mathrm{sec}$ limit, which should result in a sufficiently wide distribution to obtain reliable fits.

Figures 5(c) and (d) show results from the simulated data (e.g. Fig. 5(a)) for twelve sets of 100 time traces each for $A=1.5$ and nine sets of 100 time traces for $A=1$, where each set had a different $k_{31 \text { orig }}$ value. The CCDFs were created from these data sets and fit to various distributions (Fig. 5(b)) using MLE. As expected, the Weibull distribution produced the best fit, and the returned fit values were within error of the predicted values. In particular, when using the Weibull rate $k(t)$ as a transition rate, the returned $A$ values are very close to the expected value, and the fit of the $\lambda$ vs $k_{31 \text { orig }}$ values results in a linear relationship with a slope close to one. This was accurate for both values of $A$ used in simulations. However, the accuracy in the returned A values decreased as the $k_{31 \text { orig }}$ increased (Fig. $5(\mathrm{~d})$ ), which is likely due to the non-zero integration time (100 $\mathrm{ms}$ ) dictated by our experimental settings and thus used in the simulation. When $k_{31 \text { orig }}$ is large, the predicted "off" times become small, introducing rounding errors due to integration time. This skews the distribution and reduces accuracy in the fit values.

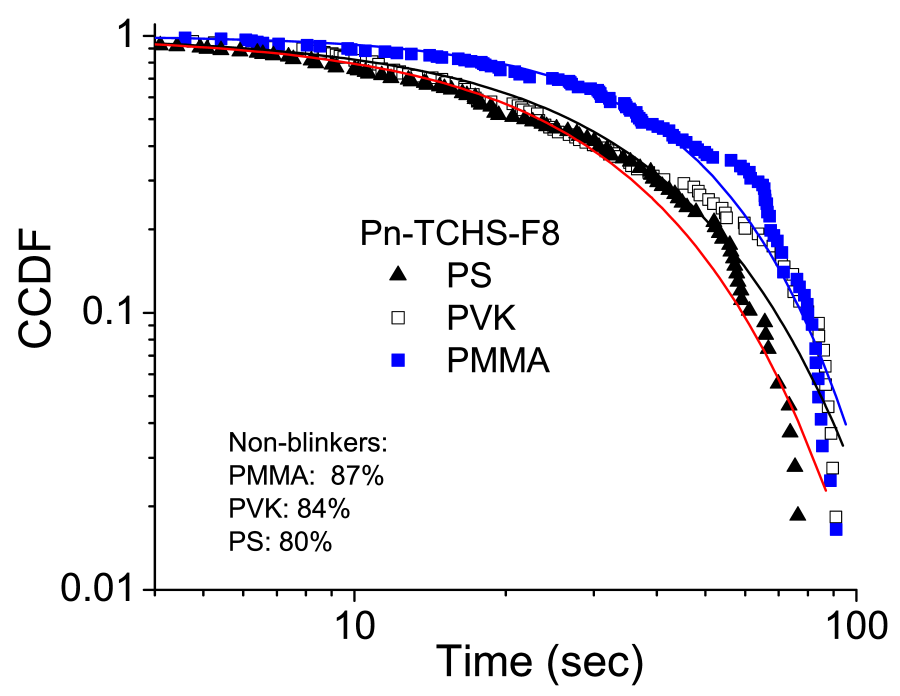

Figure 6. The complementary cumulative distribution functions (CCDFs) obtained from experimental data for PnTCHS-F8 in three different polymer host matrices: PMMA, PVK, and PS. Weibull fits are also shown. 


\subsection{Single molecule spectroscopy: experiments}

The CCDFs for the "on" times obtained from single-molecule fluorescence time trajectories compiled for small ensembles (85-105 molecules) of Pn-TCHS-F8 incorporated at ultra-low concentrations in PMMA, PVK, and PS host matrices are shown in Fig. 6. In all matrices, the data are not single-exponential, indicative of distributions in the transition rates $k_{23}$. The Weibull fits to these data produced $\lambda(A)$ values of $47.2 \mathrm{sec}(1.67), 35.8 \mathrm{sec}(1.27)$, and $31 \mathrm{sec}$ (1.29) in PMMA, PVK, and PS, respectively. The corresponding average "on" times are then $42 \mathrm{sec}, 35$ sec, and $29 \mathrm{sec}$, respectively, which are consistent with differences in oxygen permeability and diffusion coefficients in these matrices, with, for example, PMMA having these parameters lower than PS by more than an order of magnitude, which renders it a much better matrix for single-molecule fluorescence spectroscopy. ${ }^{21}$ Additionally, the percentage of molecules that exhibit blinking (i.e. reversible as opposed to irreversible degradation at the 100 sec time scale of the experiment) increased from $13 \%$ in PMMA to $20 \%$ in PS. This suggests that even though the reaction of the Pn-TCHS-F8 molecule with singlet oxygen is more probable in PS (as manifested through shorter average "on" times) as compared to PMMA, the EPO conversion back to the parent Pn-TCHS-F8 molecule is also more probable in PS, possibly due to enhanced oxygen diffusion in PS that could promote singlet oxygen quenching. Similar effect was observed when IF-TIPS molecules were added to the PMMA, serving as singlet oxygen quenchers. ${ }^{3}$

\section{CONCLUSION}

In summary, we observed dependence of photodegradation of functionalized fluorinated Pn derivatives Pn-R-F8 on the side groups $\mathrm{R}$ and local environment. More than an order of magnitude enhancement in photostability was observed in Pn-R-F8 solutions in benzene as compared to chlorobenzene; in both solutions, partial reversal of photodegradation could be achieved by thermolysis. In Pn-R-F8:PMMA films, addition of IF-TIPS molecules which served as quenchers for singlet oxygen enhanced the reversal, which was observed at room temperature in air. On the single molecule level, photostability of individual Pn-R-F8 molecules depended on the polymer matrix, with highest (lowest) photostability observed in PMMA (PS), in accordance with their oxygen permeability and diffusion coefficient characteristics. Computational modeling has provided insight into the experimental design for future single molecule fluorescence spectroscopy experiments so that the fits to the experimental data yield reliable values for transition rates involved in the model. Our experiments indicate that the choice of the side group $\mathrm{R}$ can enhance photostability by at least a factor of 2 , with the additional enhancement of more than an order of magnitude provided by the suitable choice of the surrounding environment. Several routes for reversibility of photodegradation, including addition of singlet oxygen quenchers, are also available. These fundings inform us of the molecular design considerations and conditions needed to prevent degradation of organic materials for the use in future (opto)electronic devices.

\section{ACKNOWLEDGMENTS}

We thank Dr. R. Grollman and K. Bilty for their contributions to the initial stages of this work. The National Science Foundation (DMR-1207309) and Oregon State University (OSU) URISC program (M. Li) are acknowledged for financial support. N. Q. thanks the OSU Graduate School for the Graduate Travel Award to present this work.

\section{REFERENCES}

[1] Ostroverkhova, O., "Organic optoelectronic materials: Mechanisms and applications," Chemical Reviews 116, 13279 - 13412 (2016).

[2] Fudickar, W. and Linker, T., "Why triple bonds protect acenes from oxidation and decomposition," Journal of American Chemical Society 134, 15071-15082 (2012).

[3] Grollman, R., Quist, N., Robertson, A., Rath, J., Purushothaman, B., Haley, M. M., Anthony, J. E., and Otroverkhova, O., "Single-molecule level insight into nanoscale environment-dependent photophysics in blends," Journal of Physical Chemistry C 121, 12483-12494 (2017). 
[4] Shepherd, W. E. B., Grollman, R., Robertson, A., Paudel, K., Hallani, R., Loth, M. A., Anthony, J. E., and Ostroverkhova, O., "Single-molecule imaging of organic semiconductors: Toward nanoscale insights into photophysics and molecular packing," Chemical Physics Letters 629, 29-35 (2015).

[5] Shepherd, W. E. B., Platt, A. D., Hofer, D., Ostroverkhova, O., Loth, M., and Anthony, J. E., "Aggregate formation and its effect on (opto)electronic properties of guest-host organic semiconductors," Applied Physics Letters 97, 163303 (2010).

[6] Shepherd, W. E. B., Platt, A. D., Kendrick, M. J., Loth, M., Anthony, J. E., and Ostroverkhova, O., "Energy transfer and exciplex formation and their impact on exciton and charge carrier dynamics in organic films," Journal of Physical Chemistry Letters 2, 362-366 (2011).

[7] Kendrick, M. J., Neunzert, A., Payne, M. M., Purushothaman, B., Rose, B. D., Anthony, J. E., Haley, M. M., and Ostroverkhova, O., "Formation of the donor-acceptor charge-transfer exciton and its contribution to charge photogeneration and recombination in small molecule bulk heterojunctions," Journal of Physical Chemistry C 116, 18108-18116 (2012).

[8] Ha, T. and Tinnefeld, P., "Photophysics of fluorescent probes for single-molecule biophysics and superresolution imaging," Annual Review of Physical Chemistry 63, 595-617 (2012).

[9] Riley, E. A., Bingham, C., Bott, E. D., Kahr, B., and Reid, P. J., "Two mechanisms for fluorescence intermittency of single violamine r molecules," Physical Chemistry Chemical Physics 13, 1879-1887 (2011).

[10] Hess, C. M., Riley, E. A., and Reid, P. J., "Dielectric dependence of single-molecule photoluminescence intermittency: Nile red in poly(vinylidene fluoride)," Journal of Physical Chemistry B 118, 8905-8913 (2014).

[11] Mitsui, M., Unno, A., and Azechi, S., "Understanding photoinduced charge transfer dynamics of single perylenediimide dyes in a polymer matrix by bin-time dependence of their fluorescence blinking statistics," Journal of Physical Chemistry C 120, 15070-15081 (2016).

[12] Wustholz, K. L., Bott, E. D., Isborn, C. M., Li, X., Kahr, B., and Reid, P. J., "Dispersive kinetics from single molecules oriented in single crystals of potassium acid phthalate," Journal of Physical Chemistry C 111, 9146-9156 (2007).

[13] Wong, N. Z., Ogata, A. F., and Wustholz, K. L., "Dispersive electron-transfer kinetics from single molecules on $\mathrm{TiO}_{2}$ nanoparticle films," Journal of Physical Chemistry C 117, 21075-21085 (2013).

[14] Bott, E. D., Riley, E. A., Kahr, B., and Reid, P. J., "Proton-transfer mechanism for dispersed decay kinetics of single molecules isolated in potassium hydrogen phthalate," ACS Nano 3, 2403-2411 (2009).

[15] Maenosono, S., "Monte-carlo simulations of photoinduced fluorescence enhancement in semiconductor quantum dot arrays," Chemical Physics Letters 405, 182-186 (2005).

[16] Paudel, K., Johnson, B., Neunzert, A., Thieme, M., Purushothaman, B., Payne, M. M., Anthony, J. E., and Ostroverkhova, O., "Small-molecule bulk heterojunctions: Distinguishing between effects of energy offsets and molecular packing on optoelectronic properties," Journal of Physical Chemistry C 117, 24752-24760 (2013).

[17] Paudel, K., Johnson, B., Thieme, M., Haley, M., Payne, M. M., Anthony, J. E., and Ostroverkhova, O., "Enhanced charge photogeneration promoted by crystallinity in small-molecule donor-acceptor bulk heterojunctions," Applied Physics Letters 105, 043301 (2014).

[18] Coppo, P. and Yeates, S. G., "Shining light on a pentacene derivative: The role of photoinduced cycloadditions.," Adv. Matter 17 (24), 3001-3005 (2005).

[19] Quist, N., Grolllman, R., Rath, J., Robertson, A., Haley, M., Anthony, J., and Ostroverkhova, O., "Single molecule-level study of donor-acceptor interactions and nanoscale environment in blends," Proc. of SPIE 10101-101010K (2017).

[20] Riley, E. A., Hess, C. M., Whitham, P. J., and Reid, P. J., "Beyond power laws: A new approach for analyzing single molecule photolminescence intermittency," The Journal of Chemical Physics 136, 184508 (2012).

[21] Piwoński, H., Sokolowski, A., and Waluk, J., "In search for the best environment for single molecule studies: Photostability of single terrylenediimide molecules in various polymer matrices," Journal of Physical Chemistry Letters 6, 2477-2482 (2015). 